\title{
Study on Resonant Wireless Power transmission System with Dual Transmitters
}

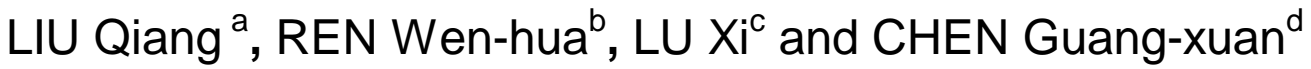

Big data Key Laboratory of the Ministry of Public Security, Zhejiang Police College, No. 555 Bin

Wen, Binjiang District, Hangzhou, 310053, China

anavylq@126.com, ${ }^{b}$ renwenhua@zjjcxy.cn, ${ }^{c} 389295554 @ q q . c o m,{ }^{d}$ chenguangxuan@zjjcxy.cn

Keywords: Wireless power transmission, dual-transmitter, resonance coupling, transmission efficiency, transmission power.

Abstract. Since the transmission efficiency and the transmission power of the existing resonant wireless power transmission (WPT) system with a single transmitter were lower, a compact resonant WPT system with dual transmitters was studied. There was a close coupling relationship between the dual transmitters of the system. Each transmitter was provided a transmitting coil. The one of the two transmitting coil was located inside the other. The equivalent circuit model of the system was established. The arithmetic expressions of the transmission efficiency and the transmission power of the system were given. The resonant frequency relationship of the transmitters was given. And the system was compared with the existing single-transmitter system. A set of resonant WPT system was produced. And the related experiments were made. The theoretical analysis and experimental research show that the transmission efficiency and the transmission power of the system are higher. This provides a useful choice for the WPT of high power and high energy.

\section{Introduction}

The resonant Wireless Power Transmission system(WPT) always with a single transmitter and a single receiver is suitable for medium-distance transmission, which depending on the frequency of the resonator to implement wireless energy transmission. The efficiency and power of RWPT is relatively low, so how to improve the efficiency and power is becoming a hot issue.

Some methods may be adopt to improve transmission efficiency and transmission power, such as trunk coil [1], coil's loss reduction, superconducting technology[2], multi-receiver[3] and so on. The trunk coil may occupy a certain space, so the application of the system may be limited. The coil's loss reduction is limited to the coil material. The cost with superconducting technology is high. And with multi-receiver, there is no coupling between multi-receivers, or the coupling coefficient is small enough to be negligible.

Under the system volume substantially the same premise, a compact dual-transmitter can be applied to the resonant wireless energy transmission system, which may improve the transmission efficiency and transmission power. Firstly, the equivalent circuit model of the system is given, then the transmission efficiency and transmission power of dual-transmitter resonant WPT system are analyze. Finally, a experimental apparatus is designed to test the feasibility. The experiment result shows the dual-transmitter resonant WPT system having a better performance rather than single-transmitter system.

\section{System Structure}

The dual emission resonance wireless power transmission system consists of electromagnetic launch systems and electromagnetic receiving system, which structure diagram shown in Fig. 1.

In Fig. 1, the transmitter no. 1 consists of coil $L_{1}$, capacitor $C_{1}$, inverter no. 1 and so on, the transmitter no. 2 consists of coil $L_{2}$, capacitor $C_{2}$, inverter no. 2 and so on, and the .inverter no. 1 and no. 2 are supplied by DC power. The electromagnetic receiving system is a single receiver, which includes the single coil $L_{3}$, the capacitor $C_{3}$ and the load posed $R_{L}$. In the system, $D$ is the distance 
between two transmitter coils $L_{1}, L_{2}$, and the receiver coil $L_{3}$. In order to minimize the space of the system, the transmitter coil is placed inside the transmitter coil. Since the distance between the two transmitting coils is very short, it has a tighter coupling relationship between the two transmitters.

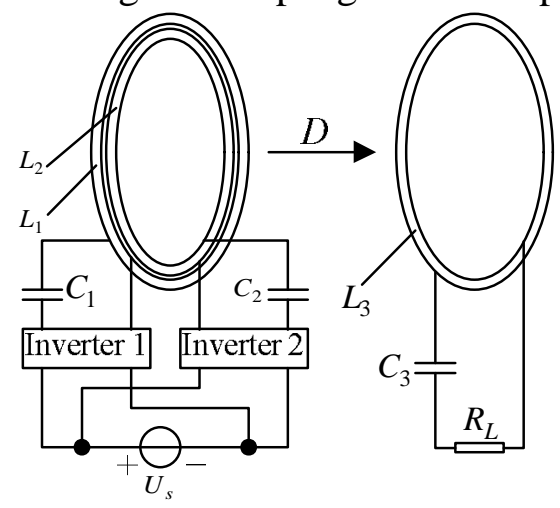

Fig. 1. Diagram of resonant WPT system with dual transmitters.

\section{Theoretical Analysis}

According to Fig. 1, the equivalent circuit model of dual-transmitter resonance WPT sytem can be derived, shown in Fig. 2. In Fig. 2, $R_{1}$ and $R_{2}$ are respectively the resistance of transmitter coil $L_{1}$ and $L_{2}, R_{3}$ is the resistance of receiver coil $L_{3} . M_{12}$ is the mutual inductance between transmitter coil $L_{1}$ and $L_{2} . M_{13}, M_{23}$ are respectively the mutual inductance between transmitter coil $L_{1}, L_{2}$ and receiver coil $L_{3} . U_{s 1}, L_{s 2}$ are respectively the equivalent voltage of the two transmitter loops, and $R_{s 1}, R_{s 2}$ are the equivalent inner resistance.

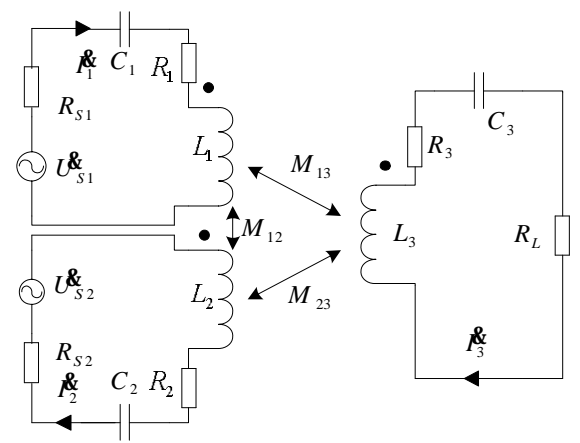

Fig. 2. Equivalent circuit model of resonant WPT system with dual transmitters.

In Fig. $1, \frac{\alpha}{\alpha}, \frac{\alpha}{2}$, and $\alpha_{3}^{\alpha}$ are respectively the current flow through the reception loop and two emission loops. According to Kirchhoff's voltage law, it is easy to obtain the Eq.,

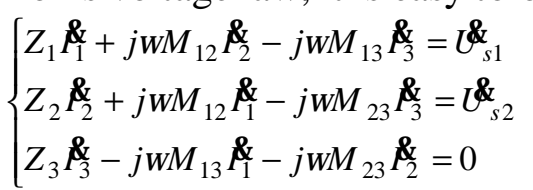

Where:

$$
\left\{\begin{array}{l}
Z_{1}=R_{s 1}+R_{1}+j\left[\omega L_{1}-1 /\left(\omega C_{1}\right)\right] \\
Z_{2}=R_{s 2}+R_{2}+j\left[\omega L_{2}-1 /\left(\omega C_{2}\right)\right] \\
Z_{3}=R_{L}+R_{3}+j\left[\omega L_{3}-1 /\left(\omega C_{3}\right)\right]
\end{array}\right.
$$

In order to simplify the analysis, it is assumed that the component parameters of two emission loops are identical, and the mutual inductance between the two emission coils $L_{1}, L_{2}$, and the reception coil $L_{3}$ are identical, then $U_{s 1}^{\&_{1}}=U_{s 2}^{\&_{2}}=U_{s}^{\&}, R_{s 1}=R_{s 2}, C_{1}=C_{2}, L_{1}=L_{2}, R_{1}=R_{2}, M_{13}=M_{23}=M$, and the current $\alpha_{1}^{\alpha}, f_{2}^{\alpha}$, and $f_{3}^{\alpha}$ can be derived from Eq. (1) as Eq. (3). The Eq. (3) show that the load current $\mathcal{F}_{3}$ being related to the power frequency, the mutual inductance between the transmitter coil and the receiving coil, the mutual inductance between the two transmitter coils. 


$$
\left\{\begin{array}{l}
\not_{1}^{\alpha}=\not_{2}^{\alpha}=\frac{Z_{3} \&_{s 1}}{\left(Z_{1}+j \omega M_{12}\right) Z_{3}+2 \omega^{2} M^{2}} \\
\&_{3}^{\alpha}=\frac{j 2 \omega M U_{s 1}^{\&_{s}}}{\left(Z_{1}+j \omega M_{12}\right) Z_{3}+2 \omega^{2} M^{2}}
\end{array}\right.
$$

In order to obtain maximum value of load current $\frac{\alpha}{3}$, the natural frequency $f_{1}, f_{2}$ of transmitter coil no. 1 and no.2, and the natural frequency $f_{3}$ of receiving coil are equal to the working frequency of power. Here, the relationship of $f_{1}, f_{2}$ and $f_{3}$ is given by

$$
\left\{\begin{array}{l}
f_{1}=f_{2}=1 /\left(2 \pi \sqrt{\left(L_{1}+M_{12}\right) C_{1}}\right) \\
f_{3}=1 /\left(2 \pi \sqrt{L_{3} C_{3}}\right)
\end{array}\right.
$$

As the Eq. (4) showed above, the natural frequency of two transmitter coils is related to the inductance and capacitance of transmitter coil, and also the mutual inductance $M_{12}$ between the two transmitter coils.Based on the Fig. 2, the input power $P_{\text {in }}$ of two transmitter loops and the power $P_{\text {out }}$ of load $R_{L}$ in receiving loop can be calculated as

$$
\left\{\begin{array}{l}
P_{\text {in }}=I_{1} U_{s 1}+I_{2} U_{s 2}=2 I_{1} U_{s 1} \\
P_{\text {out }}=I_{3}^{2} R_{L}
\end{array}\right.
$$

According to the calculating formula of transmitting efficiency $\eta$ and the Eq. (3) and Eq. (5), the transmitting efficiency $\eta$ and transmitting power $P_{\text {out }}$ can be calculated as

$$
\left\{\begin{array}{l}
\eta=\frac{\omega^{2} M^{2} R_{L}}{\mid Z_{3}\left[Z_{3}\left(Z_{1}+j \omega M_{12}\right) / 2+\omega^{2} M^{2}\right]} \\
P_{\text {out }}=\frac{\omega^{2} M^{2} U_{S 1}^{2} R_{L}}{\left|\left[Z_{3}\left(Z_{1}+j \omega M_{12}\right) / 2+\omega^{2} M^{2}\right]^{2}\right|}
\end{array}\right.
$$

When the system is working in resonance state, that is the natural frequency of two transmitters and one receiver conform to the Eq. (4), then the the transmitting efficiency $\eta$ and transmitting power $P_{\text {out }}$ can be calculated as

$$
\left\{\begin{array}{l}
\eta=\frac{\omega^{2} M^{2} R_{L}}{\left(R_{L}+R_{3}\right)\left[\left(R_{L}+R_{3}\right)\left(R_{s 1}+R_{1}\right) / 2+\omega^{2} M^{2}\right]} \\
P_{\text {out }}=\frac{\omega^{2} M^{2} U_{s 1}^{2} R_{L}}{\left[\left(R_{L}+R_{3}\right)\left(R_{s 1}+R_{1}\right) / 2+\omega^{2} M^{2}\right]^{2}}
\end{array}\right.
$$

If we remove the second transmitter in Fig. 2, we can get the single transmitter resonant WPT system. Then the transmitting efficiency $\eta_{1}$ and transmitting power $P_{1}$ under resonance state can be calculated as

$$
\left\{\begin{array}{l}
\eta_{1}=\frac{\omega^{2} M^{2} R_{L}}{\left(R_{L}+R_{3}\right)\left[\left(R_{L}+R_{3}\right)\left(R_{s 1}+R_{1}\right)+\omega^{2} M^{2}\right]} \\
P_{1}=\frac{\omega^{2} M^{2} U_{s 1}^{2} R_{L}}{\left[\left(R_{L}+R_{3}\right)\left(R_{s 1}+R_{1}\right)+\omega^{2} M^{2}\right]^{2}}
\end{array}\right.
$$

where, the natural frequency $f_{10}$ of transmitter in the single transmitter system and the natural frequency $f_{30}$ of receiver can be computed as,

$$
\left\{\begin{array}{l}
f_{10}=1 /\left(2 \pi \sqrt{L_{1} C_{10}}\right) \\
f_{30}=1 /\left(2 \pi \sqrt{L_{3} C_{3}}\right)
\end{array}\right.
$$

here, $C_{10}$ is the matched transmitter capacitor of the single transmitter system, and the capacitor of $C_{10}$ is different from the capacitor of $C_{1}$ in dual transmitter system.

According to the Eq. (7) and Eq. (8), the ratio $\eta / \eta_{1}$ and $P_{\text {out }} / p_{1}$ can be calculated as 


$$
\left\{\begin{array}{l}
\frac{\eta}{\eta_{1}}=\frac{\left(R_{L}+R_{3}\right)\left(R_{s 1}+R_{1}\right)+\omega^{2} M^{2}}{\left(R_{L}+R_{3}\right)\left(R_{s 1}+R_{1}\right) / 2+\omega^{2} M^{2}} \\
\frac{P_{\text {out }}}{P_{1}}=\left[\frac{\left(R_{L}+R_{3}\right)\left(R_{s 1}+R_{1}\right)+\omega^{2} M^{2}}{\left(R_{L}+R_{3}\right)\left(R_{s 1}+R_{1}\right) / 2+\omega^{2} M^{2}}\right]^{2}
\end{array}\right.
$$

From the Eq. (10), it's easy to see that the transmitting efficiency and power of dual-transmitter system are greater than those of single-transmitter system. That is to say the transmitting efficiency and power being increased. In addition, the resonant frequency of the dual-transmitter system is different from the single-transmitter system. The former is largely related to the mutual inductance $M_{12}$ between two transmitter coils, but the latter is not related to the mutual inductance $M_{12}$.

\section{Experimental results}

In order to verify the efficiency and power characteristics of dual-transmitter resonator wireless energy transmission system, a resonant WPT experimental system was established, shown in Fig. 3.

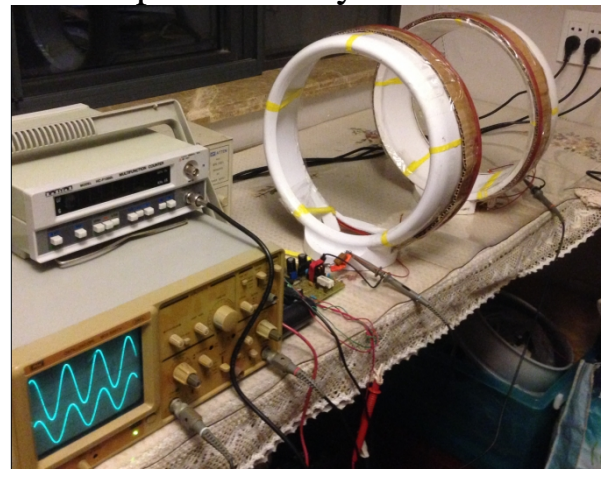

Fig. 3. Resonant WPT experiment system.

In the experimental system, there are two half-bridge inverters supplied by a DC power, two transmitter coils, two transmitter capacitors, a receiver coil, and a receiving capacitor.We choose the physical parameters of the system as follows, the diameters of coil $L_{1}$ and $L_{2}$ are respectively $315 \mathrm{~mm}$ and $305 \mathrm{~mm}$, the diameter of coil $L_{3}$ is $315 \mathrm{~mm}, L_{1}=L_{2} \approx 14.7 \mu \mathrm{H}, L_{3} \approx 14.7 \mu \mathrm{H}, M_{12} \approx 9.25 \mu \mathrm{H}, R_{L}=30 \Omega$, $R_{s 1}=R_{s 2} \approx 3.0 \Omega, R_{1}=R_{2} \approx 0.141 \Omega, R_{3} \approx 0.141 \Omega, C_{1}=C_{2}=1 \mathrm{nF}, C_{3}=1.8 \mathrm{nF}, C_{10}=1.8 \mathrm{nF}, U_{s 1}=U_{s 2} \approx 8 \mathrm{~V}$. Based on the parameters, it is easy to calculate the coupling coefficient between the two transmitting coils about 0.63 .

In the experiment, the central axis of the transmitter and receiver coils are kept in a straight line.In the case of fixed the resonance frequency $(0.827 \mathrm{MHz})$, the distance between the receiver coil and the transmitter coil is adjusted from near to far, and the measurement results shown in Fig. 4.

In Fig. 4, the curve shows the relationship between transmission efficiency and transmission distance of dual-transmitter system and single-transmitter system. Both the theoretical transmission efficiencies of dual-transmitter and single-transmitter system growth over transmitting distance are reduced, but the experimental values growth over transmission distance firstly increase and then decrease. The experimental value of transmission efficiency for dual-transmitter sytem reaches the maximum 0.571 at the disctance about $10 \mathrm{~cm}$, and corresponding theoretical value is 0.619 . The experimental value of transmission efficiency for single-transmitter sytem reaches the maximum 0.468 at the disctance about $9 \mathrm{~cm}$, and corresponding theoretical value is 0.522 . So both the theoretical and experimental transmission efficiency of dual-transmitter sytem may have a performance improvement about $10 \%$ rather than single-transmitter system. In addition, the theoretical value and the experimental value of the pace of change in the transmission efficiency of the two systems are inconsistent, which is consistent with the rusults of reference [4-5]. And this is a frequency splitting performance caused by the over-coupling between the transmitter and the receiver.

In Fig. 4, the curve shows the relationship between transmission power and transmission distance of dual-transmitter system and single-transmitter system. Both the theoretical transmission efficiencies of 
dual-transmitter and single-transmitter system growth over transmitting distance firstly increase and then decrease, and the pace of change is basically consistent with each other. The experimental value and theoretical value of transmission efficiency for dual-transmitter sytem reache the maximum value at the disctance about $10 \mathrm{~cm}$, and corresponding values are respectively 9.53 Watt and 8.75 Watt. But for the single-transmitter system, the maximum value point is about at $9 \mathrm{~cm}$, and the corresponding theoretical value and experimental value are respectively 5.05 Watt and 4.46 Watt. So both the theoretical and experimental transmission efficiency of dual-transmitter sytem may have a performance improvement about $80 \%$ rather than single-transmitter system.
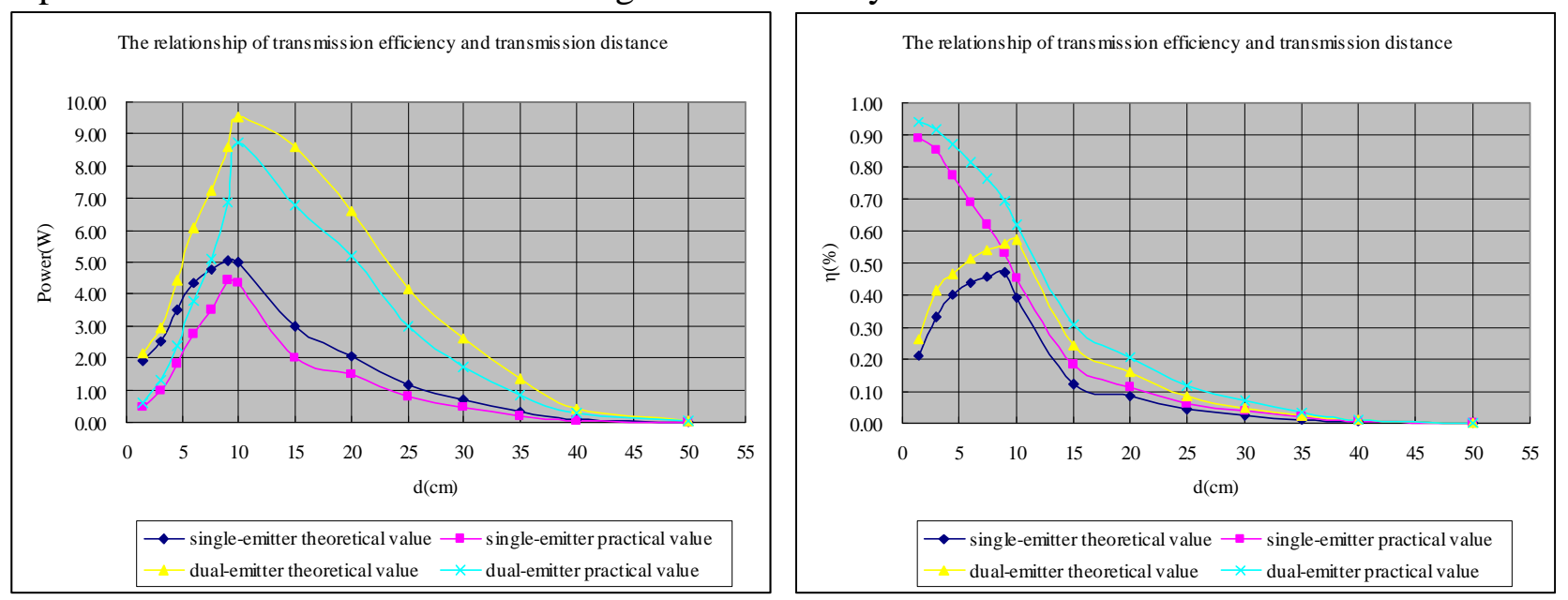

Fig. 4. The experiment curve of dual-emitter resonant WPT system.

In addition, both the transmission power of dual-transmitter and single-transmitter system growth over transmitting distance firstly increase and then decrease. And it also reflects the frequency splitting performance caused by the over-coupling between the transmitter and the receiver .

\section{Conclusion}

From the theoretical analysis and experimental results above, it may be easily found that both the transmission efficiency and transmission power have a considerable performance improvement with tight-coupling dual-transmitter WPT system rather than single-transmitter system. Unfortunately, there is also a frequency splitting performance in the dual-transmitter system, which exists in the single-transmitter system. In future research, we need to pay attention to the issue.

\section{Acknowledgements}

This work was financially supported by the National Undergraduate Training Programs for Innovation and Entrepreneurship National Project (201411483008), Zhejiang Provincial Education visiting scholar Project(FX2014123), Zhejiang Provincial Education Department general research project (Y201533572) and Zhejiang Police College Research Project (20150616).

\section{References}

[1] M. Kiani, J. Uei-Ming and M. Ghovanloo. IEEE Trans. on Biomedical Circuits and Systems Vol. 5 (2011), p. 579-591.

[2] D. W. Kim, Y. D. Chung and H. K. Kang. IEEE Trans. on Applied Superconductivity Vol. 22 (2012), p. 5400604-5400604.

[3] A. Kurs, R. Moffatt. Applied Physics Letters Vol. 96 (2010), p. 044102-044102-3.

[4] A. P. Sample, D A Meye. IEEE Trans. on Industrial Electronics Vol. 58 (2011), p. 544-554.

[5] X. Zhang, Q. X. Yang, H. Y. Chen, et al. Proceedings of the CSEE Vol. 32 (2012), p. 167-173. 